\title{
MedienPädagogik
}

Zeitschrift für Theorie und Praxis der Medienbildung

www.medienpaed.com

ISSN 1424-3636

Jahrbuch Medienpädagogik 15:

Erziehungswissenschaftliche und medienpädagogische Online-Forschung:

Herausforderungen und Perspektiven

Herausgegeben von Johannes Fromme, Stefan Iske, Therese Leik, Steffi

Rehfeld, Jasmin Bastian, Manuela Pietraß und Klaus Rummler

\section{Understanding Digital Media}

\section{Thesen zu ethnografischen Verfahren im Hinblick auf die Online-Forschung}

Dan Verständig und Jens Holze

\section{Zusammenfassung}

Ausgehend davon, dass digitale Medien konstitutiv für soziale und kulturelle Räume sind, thematisiert der Beitrag thesenartig zwei zentrale Aspekte der erziehungswissenschaftlichen Online-Forschung. Zum einen wird die Bedeutung von onlineethnografischer Forschung im Horizont digitaler Medialität diskutiert, die als eine Methodologie den Blick auf die Formstrukturen von digitalen Medien und deren kulturellen und sozialen Rahmungen ermöglicht. Zum anderen erfordert der Umgang mit digitalen Daten eine methodische und methodologische Reflexivität hinsichtlich ihrer strukturellen Einbettung und Beschaffenheit. Hierbei wird gezielt auf die Frage abgezielt, wie man mit den technologischen Infrastrukturen überhaupt umgeht und welche Rolle nicht nur Daten, sondern auch digitale Methoden für die Erziehungswissenschaft spielen. Beide Thesen stehen in einer Wechselbeziehung. Es geht im Beitrag darum, die Verschränkungen deutlich zu machen und für einen reflexiven Umgang mit digitalen Daten, Methoden und Methodologien zu plädieren. 


\title{
Understanding Digital Media - 10 Theses on Ethnographic Methods with regard to Online Research
}

\begin{abstract}
Based on the fact that the digital media are constitutive for social and cultural spaces, the contribution establishes two theses on educational online research. On the one hand, the importance of online ethnographic research in the horizon of digital mediality is discussed, which, as a methodology, enables a view of the formal structures of digital media and their cultural and social frameworks. On the other hand, the handling of digital data requires a methodological and methodological reflexivity with regard to their structural embedding and nature. This thesis specifically adresses the question of how to deal with technological infrastructures, and how to think about not only data, but also digital methods for educational science. Both theses are interrelated, where the point is to make the entanglements visible and to plead for a reflexive use of digital data, methods and methodologies.
\end{abstract}

\section{Einleitung}

«We shape our tools and thereafter our tools shape us»

Mit diesen Worten fasste einst John Culkin (1967, 70), ein Freund und Kollege Marshall McLuhans, dessen Einsichten zusammen. Wir Menschen beschreiben uns über unseren Fortschritt und über unsere Technologien, die uns diesen Fortschritt ermöglichen und zugleich ein Motor für weitere Entwicklungen sein können. Es ist daher keine neue These, dass sich Gesellschaften über Technologien definieren und an Optimierungsprozessen messen. Das Internet kann dabei als zeitgenössische Technologie begriffen werden, die massgeblich auf diese Selbstbeschreibungsprozesse einwirkt. Die Online-Forschung bietet hier einen möglichen Zugang zur Deutung der Prozesse und theoretischen Rückbindung, sie ist jedoch gleichzeitig ganz grundlegend von der rasanten Entwicklung des Netzes einerseits und hohen 
Dynamik der forschungsmethodischen und methodologischen Fragen andererseits geprägt (vgl. Consalvo und Ess 2011; Dutton 2014). Trotz der hohen Entwicklungsdynamik und Heterogenität der jeweiligen sozialen, kulturellen und politischen Ausprägungsformen des Digitalen lässt sich ein gemeinsamer Nenner bestimmen. Es ist der Code (vgl. Lessig 1999), der diesen digitalen Architekturen zu Grunde liegt und die Infrastruktur sowie die immer komplexer werdende «Algorithmizität» (Stalder 2016) umspannt.

Entgegen bisheriger Bestrebungen im Rahmen einer erziehungswissenschaftlich fundierten Online-Forschung, die sich in erster Linie vorwiegend entlang der Untersuchung von Nutzungsweisen im Anschluss an digitale und vernetzte Technologien verorten lassen, soll es in diesem Beitrag darum gehen, die Infrastruktur des Netzes aus einer strukturtheoretischen Perspektive in Anlehnung an Jörissen und Marotzki (2009) sowie Jörissen $(2014,2016)$ in den Blick zu nehmen und danach zu fragen, inwieweit die Betrachtung der digitalen Infrastrukturen helfen kann, die Qualität des Digitalen methodisch aber auch methodologisch genauer in den Blick zu nehmen.

Der Beitrag widmet sich dieser Schwerpunktsetzung entlang der Diskussion um ethnografische Verfahren Online (vgl. hierzu u.a. Marotzki 2003, 2008; Kozinets 2006, 2010) und stellt dazu zwei The$\operatorname{sen}^{1}$ auf, die in diesem Beitrag diskutiert und argumentativ dargelegt werden sollen. Ziel ist es, aus bildungstheoretischer Perspektive die Relevanz einer Online-Ethnografie, also einer Ethnografie, die in der Lage ist, soziale Phänomene vor dem Hintergrund einer digitalen Medialität zu erschliessen, zu begründen, aber auch für die Notwendigkeit einer fortlaufenden methodischen Weiterentwicklung im

1 Der Titel des Beitrags geht auf den Vortrag im Rahmen der Herbsttagung der Sektion Medienpädagogik der DGfE 2017 in Magdeburg zurück. Das Wortspiel 10 - also 2 im Binärformat - zu verwenden bietet sich eher für die Dramaturgie eines Vortrags als für diesen Aufsatz an. Es ist trotzdem noch im Beitragstitel verblieben, da die Argumentationsfigur des Textes auf die Reflexivität der Strukturen abhebt und dies eine passende Ergänzung hierzu darstellt. 
Kontext von Algorithmizität und sich verändernder digitaler Plattformen zu plädieren. Damit verfolgt der Beitrag gleichzeitig zwei Zielsetzungen. Erstens soll auf die methodische Entwicklung von ethnografischen Verfahren im Netz abgezielt werden und danach gefragt werden, wie sich Ethnografie zu digitalen medialen Artefakten verhält. Es geht also um Methodenentwicklung. Zweitens soll der Blick auf die oftmals unsichtbaren Strukturen gerichtet werden, die sich hinter den sichtbaren Repräsentationsformen durch digitale Medien ergeben. Es geht also um Softwarecode. Unter der Annahme, dass dieser ebenfalls als ethnografisches Artefakt verstanden werden kann, wird gefragt, welche Herausforderungen sich hieraus hinsichtlich der ethnografischen Forschung ergeben.

\section{Erste These: Digitale Medienstrukturen sind kulturell spezifisch. Ethnografische und strukturale Elemente ermöglichen die Interpretation von Bedeutung im Kulturraum Internet.}

Ausgehend vom Konzept der Strukturalen Medienbildung nach Jörissen und Marotzki (2009) kann das Internet als zugrundeliegende Plattform für einen umfassenden Prozess der Digitalisierung verstanden werden, der seit Ende des 20. Jahrhunderts beobachtet werden kann (vgl. Holze 2017; Verständig 2017). Darunter ist eine neue Stufe eines kontinuierlichen Medialisierungsprozesses zu verstehen, der als ein Phänomen der Moderne und Postmoderne neben (oder gar in Verschränkung mit) anderen Metaprozessen wie der Globalisierung betrachtet werden kann. In der daraus resultierenden digitalen Medialität (vgl. Jörissen 2014) ergeben sich qualitative Veränderungen für eine Vielzahl von Konzepten aus den Sozial- und Kulturwissenschaften und eben auch für die Erziehungswissenschaften, aus deren Perspektive heraus hier argumentiert wird. So wird angenommen, dass Medialität ein konstitutives Element von Bildung darstellt, dass Bildungsprozesse in der digitalen Medialität mit ihren jeweils anderen Formen der Vergemeinschaftung und 
Formen von digitalen Sozial- und Kulturräumen, anders gerahmt sind als in einer Welt dominanter analoger Medienarchitekturen.

\section{Medien als Umwelten}

Die Argumentation dafür geht im Kern auf den Literatur- und Medienwissenschaftler Marshall McLuhan zurück, der mit seinem Aphorismus «Das Medium ist die Botschaft» ausgehend vom Werk Harold Innis' für die Struktureffekte von Medien argumentierte und damit kurzzeitig globale Popularität erreichte, die bis heute anhält. Dabei legte er zunächst einen Medienbegriff zugrunde, der nicht an die Kommunikationsfunktion der Medien gekoppelt ist, sondern das Medium als Erweiterung oder "Ausweitung unserer eigenen Person» (McLuhan 1992, 17) versteht. McLuhans Fokus lag dabei spezifisch nicht auf Inhalten von Medien - dem Programm oder den Kommunikationsgegenständen - sondern auf der Form des Mediums und den sich daraus ergebenden strukturalen Effekten. So sei das elektrische Licht nur durch seine Anwesenheit in der Lage, dafür zu sorgen, dass der Tag/Nacht-Zyklus durchbrochen werden kann und Aktivitäten aller Art auch nachts möglich sind. Ob es sich dabei um ein Baseballspiel oder einen gehirnchirurgischen Eingriff handele, die aufgrund von elektrischer Beleuchtung ermöglicht werden, spiele eine untergeordnete Rolle. Ferner sagt er, «dass der 〈Inhalt jedes Mediums immer ein anderes Medium ist» (ebd., 18). Jedes Medium spannt demnach eine neue Umgebung auf, welche wieder zum Hintergrund für weitere Medien werden kann. In komplexen Medienumwelten beeinflussen sich die Medien so auch gegenseitig. McLuhan verweist in seinem späteren Werk immer wieder auf das gestaltpsychologische Konzept von Figur und Hintergrund: Beide sind füreinander konstitutiv, jedoch hat unsere beschränkte Sinneswahrnehmung Probleme, beides gleichzeitig in den Blick zu nehmen. Insbesondere der Hintergrund wäre im Gegensatz zur Figur nahezu immer unsichtbar: 
«The figure is what appears and the ground is always subliminal. Changes occur in the ground before they occur in the figure» (Logan 2013, 29). ${ }^{2}$

Im Verständnis McLuhans, und das scheint heute mehr denn je anschlussfähig, spannen Medien aber als solche immer neue Umgebungen auf, sie «umwelten» uns und sind konstitutiv für unser Selbst- und Weltverhältnis. Erst neue Medien und die durch sie erzeugten Umgebungen ermöglichen uns jedoch überhaupt, die vorherige Medienumgebung wahrzunehmen, weil dann der vorherige Hintergrund zur Figur vor einem neuen Hintergrund wird.

Elektrische Medien sieht McLuhan als neues mediales Paradigma, als neue Ära nach der Gutenberg-Galaxis. Den Begriff der Digitalen Medien kannte McLuhan zwar nicht, verweist aber immer wieder auf Elemente wie die aufkommende Computertechnologie und die Automatisierung. Robert K. Logan argumentiert insbesondere, dass McLuhan viele der digitalen Phänomene lange vor ihrer Entstehung prophezeit habe (vgl. Logan 2013, 153ff.). Digitalis im Lateinischen bedeutet «zum Finger gehörend» und wurde - auf das Zählen mit den Fingern verweisend - auch als 〈Ziffer〉 gedeutet. Diese Brücke zum Tastsinn wäre für McLuhan sicher ein gefundenes Fressen, vermutete er doch, dass im elektrischen Zeitalter vormals tribale Strukturen und ein allumfassender Gemeinschaftssinn (den er meist mit dem Tastsinn verband) im sogenannten audio-taktilen Raum $^{3}$, dem Gegenentwurf zu einem primär visuell dominierten Raum der Gutenberg-Galaxis, zurückgewonnen werden würden:

2 Logan bezieht sich auf ein Zitat aus privater Konversation McLuhans, das online an dieser Stelle zu finden ist: http://imfpu.blogspot. com/2008/12/magritte.html.

3 Als akustischen Raum oder Audial-Tactile Space bezeichnet McLuhan den Raum tribaler Gesellschaftsformen, dessen Reichweite und Verbreitung gleichförmig in alle Richtungen geschieht und der den Gegenentwurf zum singulären Fokus auf das Visuelle, der durch die Schrift etabliert wurde, darstellt (vgl. McLuhan 1969, 23ff.). 
«Ours is a brand-new world of allatonceness. 'Time' has ceased, 'space' has vanished. We now live in a global village...a simultaneous happening. We are back in acoustic space. We have begun again to structure the primordial feeling, the tribal emotions from which a few centuries of literacy divorced us》 (McLuhan 1967, 63).

Insofern kann man digitale Medien als den aktuell dominanten Modus elektrischer Medien begreifen, die auch alle anderen Medien erfassen und alte (analoge) Medien obsolet machen, eines der «Laws of Media» von McLuhan (vgl. McLuhan und McLuhan 1989). Dies bedeutet nicht, dass physische Bücher oder analoge Technologien wie Schallplatten, VHS oder Mikrofilm plötzlich verschwänden, aber ihre Rollen und ihre Relevanz verändern sich und sie bleiben mitunter nicht die dominanten medialen Formen, sondern wandeln sich, wie schon erläutert, vom Hintergrund zur Figur. Viele Phänomene der digitalen Medien mögen auf Ebene der Figur ähnlich aussehen, wie schon zuvor bekannte Phänomene, aber sie finden vor einem neuen Hintergrund statt, der zu einer anderen Qualität führen kann. Das ist der Wandel, vor dem sich alle Wissenschaften und insbesondere die Bildungswissenschaft mit ihren Gegenständen neu in Beziehung setzen sollten.

Wir befinden uns folglich in einer digitalen Medialität (vgl. Jörissen 2014). Vor diesem Hintergrund macht eine Unterscheidung zwischen online und offline immer weniger Sinn, vielmehr leben wir in einer von digitalen oder digitalisierten Medien durchzogene Realität, in der diese Grenzen immer mehr verschwimmen. Dies stellt auch einen neuen Ausgangspunkt für qualitative Forschungsmethoden insbesondere vor dem Konzept von Digital Humanities dar (vgl. Berry 2012). So scheint ein Fokus auf die Strukturen von Onlinediensten auf dieser Basis von steigender Bedeutung, stellen Sie doch sowohl eine Figur vor einem komplexen Hintergrund digitaler und elektrischer Medien als auch Hintergrund für konkret zu beobachtende Phänomene in einem meist komplexen Dualismus 
dar. Gleichzeitig werden die technischen Rahmenbedingungen für ein sich weiter dynamisch entwickelndes Internet immer komplexer und dessen Präsenz im Alltag kann als ubiquitär angenommen werden, wobei es aber in unterschiedlichen Formen auf einer Vielzahl von Endgeräten beheimatet ist und mannigfaltige Nutzungspraktiken hervorbringt.

\section{Mediale Umwelten als Teil von Ethnografie}

Die online-ethnografischen Strukturmerkmale nach Marotzki (2003) sind als Ergebnis einer empirischen Studie von Online-Communities entstanden und werden auch heute noch zur Strukturanalyse von Vergemeinschaftungsplattformen im World Wide Web verwendet. Diese sozio-technischen Strukturmerkmale waren und sind als Kontext für neue Handlungspraxen in Online-Gemeinschaften relevant, denn sie stellen die Kommunikations- und Partizipationsstrukturen zur Verfügung, in deren Rahmen Menschen handlungsfähig sind. Sie haben dabei durchaus Erneuerungen erfahren, die den technischen und strukturalen Entwicklungen des Internet der letzten 14 Jahre punktuell Rechnung tragen (vgl. z.B. Jörissen und Marotzki 2009). Um (sub-)kulturelle Praktiken begreifbar zu machen, die in diesen digitalen Medienumgebungen entstehen, scheint ein online-ethnografischer Ansatz insbesondere geeignet, da Praktiken, Bedeutungen und Zusammenhänge anhand von teilnehmender Beobachtung dokumentiert, interpretiert und rekonstruktiv analysiert werden können. Insbesondere stellen dabei Innovationen im Bereich der Infrastruktur ${ }^{4}$, Verknüpfungen zwischen verschiedenen Diensten und die dem Internet eigene Dynamik der Unbestimmtheit des Mediums Herausforderungen dar. Dafür benötigt ein online-ethnografischer Zugang eine steigende Flexibilität im Umgang mit einem sich immer weiter ausdifferenzierenden Internet, aber auch eventuell eine verstärkte Betrachtung von Algorithmen und Software für die Merkmale von soziographischen Strukturen oder

4 Gemeint sind hier beispielsweise Mobile Apps oder Responsive Designs, die auf unterschiedlicher Hardware und damit ganz verschiedenen Endgeräten laufen. 
Regelsystemen. Gleichzeitig entstehen auf den genannten Plattformen immer komplexere Sozialstrukturen, die von aussen fremd wirken mögen, es aber für die Beteiligten natürlich nicht sind. Ethnografisch erschliesst sich daher sowohl aus der Umgebung als auch aus den Bedeutungszuweisungen der Beteiligten, die immer kulturell gerahmt sind, die Sinnhaftigkeit, die wir erfassen und verstehen können wollen.

Insofern kann die Online-Ethnografie trotz der steigenden Popularität von Social Network Sites und Plattformen in Anschlag gebracht werden. Die Anschlussfähigkeit ergibt sich aus der Annahme, dass soziale Phänomene in verschiedenen Plattformen über die Abbildung klassischer Gemeinschaftsstrukturen systematisch in den Blick genommen werden können, wenn ein ethnografischer Blick auf soziale Phänomene im Netz eingenommen wird. Eine klare Abgrenzung kann allerdings nicht mehr auf technischer Ebene erfolgen, da sich Communities auch auf einen Verbund verschiedener Dienste erstrecken und sowohl online als auch offline organisiert sind. Was dazu auffordern kann, den Fokus der Forschung auch während des Vorgehens stets zu triangulieren und kritisch-reflexiv zu hinterfragen.

In Anlehnung an Wellman kann festgehalten werden, dass sich die erste Phase der Internet Studies primär auf ethnografische Beobachtungen gestützt hat (vgl. Wellman 2004). Daran anschliessend gehen wir davon aus, dass aufgrund der sich weiterentwickelnden technologischen Umwelten auch für das mittlerweile stark diversifizierte Netz sich weiterhin Bedarf für ethnografische Zugänge abzeichnet. Dies zeigt sich bei einer Vielzahl an Phänomenen, wir wollen es beispielhaft kurz an Let's-Play-Videos illustrieren. Hierbei handelt es sich um eine ursprünglich subkulturelle Praxis des Spielens digitaler Spiele mit/vor einem Online-Publikum entweder in aufgezeichneten Videos oder im Livestream. Let's Play als Genre 
ist beim Massenpublikum mittlerweile überaus beliebt ${ }^{5}$, Let's Player sind prominent auch in den Massenmedien präsent und einige wenige erfreuen sich eines signifikanten kommerziellen Erfolgs. Plattformen wie YouTube und Twitch haben sich auf die formalen Bedarfe dieses Genres eingestellt, das Live-Streaming des eigenen Spiels ist mittlerweile häufig fester Bestandteil schon der Spielsoftware oder der jeweiligen Plattformen. ${ }^{6}$ Die Schwelle, das eigene Spiel mit einem weltweiten Publikum zu teilen, ist insofern konstant niedriger geworden, gleichzeitig ergeben sich daraus auch Potentiale für subversive Praktiken (vgl. Verständig und Holze 2016). Die Motivation, die Praktiken und die Bedeutung bestimmter Elemente im Kontext von Let's Plays sind von der Gamer-Subkultur abhängig und nur vor diesem Hintergrund zu verstehen. So ist beispielsweise das Schreiben des Buchstaben «F》 im Livechat eines Streams eine Form der Respektsbezeugung, die auf eine entsprechende Szene des MultiplayerShooters Call of Duty: Advanced Warfare basiert.?

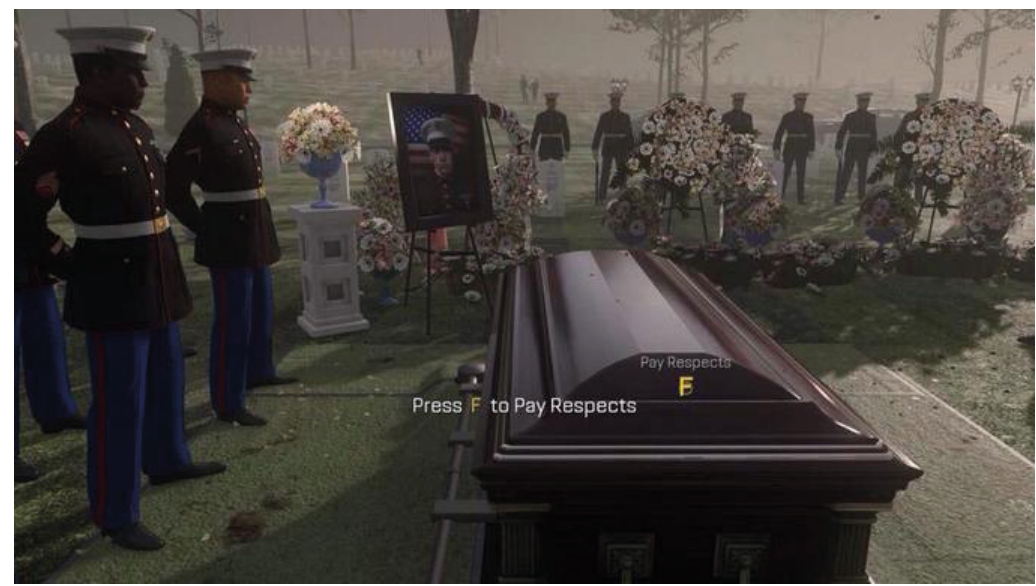

Abb. 1.: Szene aus Call of Duty: Advanced Warfare (Activision 2014). Quelle: Eigener Screenshot.

5 Drei der 25 deutschen YouTube-Kanäle mit den meisten Abonnenten sind auf Let's Plays spezialisiert, wie man der folgenden Übersicht entnehmen kann: https://socialblade.com/youtube/top/country/de/mostsubscribed.

6 Beispielsweise über Steam Broadcasting oder NVidia Shield.

7 Vgl. https://knowyourmeme.com/memes/press-f-to-pay-respects 
Solche und ähnlich codierte Praktiken lassen sich meist nicht aus dem jeweiligen Kontext heraus verstehen, denn die Praktik ist nicht länger an das konkrete Spiel gebunden, sondern hat sich als Meme rekontextualisiert. Damit wird deutlich, dass - ähnlich zu vielen anderen ethnografischen Beobachtungen - viele scheinbar oberflächliche Praktiken auf komplexe kulturell verschlüsselte Bedeutungen verweisen können. Hier wird ebenfalls deutlich, dass technologische Elemente für das Verstehen ebenfalls von zentraler Bedeutung sein können. Dies ist lediglich ein Beispiel aus der Gaming-Subkultur, das den generellen Mechanismus der Rekontextualisierung von Bedeutung in digitalen Medien illustrieren soll. Es macht aber deutlich, dass solche Bedeutungsgehalte oft nicht direkt aus den jeweiligen Kontexten erschlossen werden können, sondern nur kontextübergreifend verstanden werden können.

Der Umgang mit der digitalen Medialität bei der ethnografischen Kartographierung solcher Phänomene setzt also einen reflektierten Umgang mit ihr voraus. Das betrifft nicht zuletzt die grosse Sphäre der digitalen Daten, die einer derartigen Betrachtung notwendigerweise vorausgeht und die daher Gegenstand unserer zweiten These ist.

\section{Zweite These: Der Umgang mit digitalen Daten erfordert methodische und methodologische Reflexi- vität hinsichtlich ihrer strukturellen Ein- bettung und Beschaffenheit.}

Diese These ist grundsätzlich keine neue und stellt die Forschung in spezifischen Vorhaben immer wieder vor ganz individuelle Herausforderungen, weshalb eine solche These für die Online-Forschung jedoch von gesteigerter Bedeutung ist, lässt sich entlang der Qualität von digitalen Strukturen beschreiben. Dies trifft einerseits auf die konkrete mediale Ausprägung zu und andererseits wirkt sich das Digitale in seinen rekonfigurierbaren Zusammenhängen auch 
im Ganzen auf einzelne Kontexte aus, wie wir entlang der Diskussion um Digitalität und digitale Medialität bereits aufgezeigt haben. Die Analyse medialer Strukturen stellt einen geeigneten Weg dar, um mediale Phänomene zu hinterfragen und verstehend einordnen zu können. Während beim Film oder Fernsehen die Dekonstruktion des Produktionsprozesses helfen kann, ist eine solche Leistung im Hinblick auf das Digitale nur schwer möglich, scheint er gleichzeitig jedoch in irgendeiner vergleichbaren Form erforderlich. Es ist daher keine Überraschung, dass angesichts der fortschreitenden Digitalisierung mehr und mehr die Forderung nach Programmierkenntnissen im Sinne einer gestalterischen Selbstermächtigung und Hinterfragung digitaler Architekturen aufkommt.

Will man nun Online-Forschung durch eine erziehungswissenschaftliche Brille sehen, dann stellt sich die Frage, was den digitalen Medien überhaupt zugrunde liegt und wie sie sich nicht nur auszeichnen, sondern was sie erst konstituiert. Hier erscheint die von Brügger (2011) vorgenommene Unterscheidung in zwei allgemeine Ausprägungen von Web Material vor dem Hintergrund digitaler Medien recht treffend:

«It is digital and it is present on the Internet. It can therefore be considered a sub-set of, on the one hand, digital media (e.g. eprint, e-books, computer game consoles, CD-ROM/DVDs, etc.), and, on the other hand, the Internet (an infrastructure with a variety of protocols, software types, etc. - Usenet, Gopher, Internet relay chat, email, etc.)》 (ebd., 25).

Diese Unterscheidung ist deswegen hilfreich, da das Internet, als Netz der Netze mit seiner Infrastruktur, seinen vielfältigen Diensten und den zugrunde liegenden Protokollen analytisch in den Blick genommen wird. Die Infrastruktur wird insbesondere in medienpädagogisch orientierter Online-Forschung in erster Linie so verstanden, als dass es um die Betrachtung von Nutzungs- und Anwendungskontexten, also um Umgangsweisen und Praktiken im Anschluss an die Technologie geht, bisher jedoch abgesehen von 
wenigen Ausnahmen danach gefragt wurde, wie sich die digitalen Technologien in soziale, kulturelle und politische Kontexte bereits aufgrund ihrer der Technologie eingeschriebenen Werte auszeichnen. Angesichts der Digitalisierung und der immer tiefer greifenden Verflechtung von Code, Algorithmen, Soft- und Hardware in allen gesellschaftlichen Bereichen steht die Forderung nach Medienkompetenzvermittlung vor einer besonderen Herausforderung.

Es geht schliesslich darum, Prozesse und Phänomene zu erklären, die sich einerseits der Sichtbarkeit des einzelnen entziehen. Beispielsweise dann, wenn algorithmische Selektions-, Priorisierungsoder automatische Entscheidungsprozesse und ihre Auswirkungen thematisiert werden. Andererseits tragen derartige Systeme und Prozesse nicht nur zur Komplexitätssteigerung bei, sie erfahren diese gleichzeitig und zwar dann, wenn algorithmische Strukturen Kontingenz hervorbringen und diese zugleich in irgendeiner Form operationalisieren (vgl. Crawford 2015). Dies geschieht jedoch ohne soziale Interaktion und Wertzuschreibungen, sondern in Verbindung zu sozialen, kulturellen und politischen Praktiken, wie Crawford ganz treffend auf den Punkt bringt:

«By looking beyond algorithms as fetishized objects, we can account for a wider range of actors: be it developers in cubicle farms, Twitter bots, Amazon book buyers, 4channers and Redditors. And we can also look to the ways people reverse engineer algorithms, acting in direct contestation, where the troll, the artist and the hacker become key players in an agonistic system» (ebd., 7).

Doch Programmcode entsteht nicht im sozialen Vakuum, sondern ist ebenso Bestandteil jener kulturellen und sozialen Einbettungen. Daher scheint es für die Forschung im Umgang mit digitalen Strukturen gewinnbringend zu sein, sich nicht nur mit den Phänomenen auf der Anwendungsoberfläche zu befassen, sondern auch danach zu fragen, wieso die medialen und digitalen Strukturen jene sind, die vorgefunden werden und wieso sie zu diesen geworden sind 
(vgl. Chun 2006, 3f.). Eine derartig reflexive Haltung nimmt dann nicht nur den Veränderungsprozess von alten zu neuen Medien in den Blick, sondern kann gezielt dazu beitragen, Einordnungen vorzunehmen und Sinnhaftigkeiten zu reproduzieren oder zu verstehen.

\section{Getting closer to the metal}

Die Redewendung «closer to the metal» hat ihren Ursprung in der Softwareentwicklung und bedeutet so viel, wie näher an der Hardware zu entwickeln. Man arbeitet also weniger an Skriptsprachen oder auf abstrahierten Ebenen, sondern entwickelt direkt in Abhängigkeit zum Arbeitsspeicher oder dem Prozessor der Zielgeräte, um so eine höchstmögliche Performance der Software auf der laufenden Hardware zu ermöglichen. ${ }^{8}$ Wir halten diese Perspektive in Anlehnung an Brunton und Coleman (2014) auch für die Erforschung der Komplexität der sozialen Aushandlungsprozesse auf unterschiedlichen kulturellen Ebenen für relevant, denn erst wenn man tieferliegende Mechanismen und Praktiken überhaupt offenlegt, kann man den nächsten Schritt der Deskription angehen.

«When we peel back that deepest layer of materiality, we find people and practices underneath: populations of users, and the 'superusers' who operate close to the metal in their work, including system and net administrators (sys/net admins), hackers, and spammers in complex, contingent, ambiguous relationships» (Brunton und Coleman 2014, 77).

Mit kulturellen Ebenen sind die unterschiedlichen (sub-)kulturellen Ausprägungen gemeint, die sich entlang der sozialen Arenen abzeichnen lassen. Dies kann die subversive Nutzung digitaler Plattformen sein (vgl. hierzu Verständig und Holze 2016) oder die

8 Daneben kann hier auch die emanzipatorische Einstellung beschrieben werden, sich nicht nur gegenüber dem Prozess der Softwareentwicklung zu verorten, sondern sich reflexiv zu den potenziellen Anwendungsumgebungen zu verhalten. 
unterschiedlich ausgeprägten Formen der intersubjektiven Auseinandersetzung entlang legitimierter Geltungskriterien.

Seaver (2017) plädiert in diesem Zusammenhang explizit dafür, Algorithmen als Artefakte zu verstehen, die in Verflechtung zu kulturellen Praktiken und ihrer Einbettung erst sinnstiftend rekonstruiert werden können. Dabei stelle ein ethnografisches Vorgehen ein geeignetes Verfahren dar, um in unterschiedlichen Fokussen auf die jeweiligen tieferliegenden Dynamiken hinzuweisen:

«Ethnography provides a useful orientation for entering and understanding worlds of meaning-laden practice, but conventional understandings of algorithms as defined by secret procedure suggest that ethnographic approaches are infeasible without a level of access that cannot realistically be obtained. The tactics I have laid out here are techniques for routing around that challenge; they work to enact algorithms not as inaccessible black boxes, but as heterogeneous and diffuse sociotechnical systems, with entanglements beyond the boundaries of proprietary software» (ebd., 10).

Algorithmen können demzufolge nicht nur in Kultur, sondern vielmehr auch selbst als Kultur verstanden werden (vgl. ebd., 4). Code ist also ein ethnografisches Artefakt. Damit gemeint ist nicht nur die Software, die schliesslich die Anwendung zur Sichtbarkeit verhilft, sondern schon der Quellcode, der dieser Anwendung zugrunde liegt. Code ist nicht gesetzt, er entsteht im Zusammenspiel von Konzept, Design und Kultur (vgl. Jörissen und Verständig 2016). Daher ist der Umgang mit Code bei der Online-Forschung zumindest immer implizit ein Thema. Das Spektrum der Fokussierung ist dabei recht hoch, es kann die funktionale Leistung von Quellcode betreffen, die Kommentare im Quellcode, die Metadaten um ein Projekt herum oder das Verhältnis von laufender Softwareanwendung und implementierter Idee bzw. Konzeption. Gerade der letzte Aspekt ist von gesteigerter Bedeutung und zugleich herausfordernd, da Algorithmen im Feld eben nicht das sind, was man im Lehrbuch lesen 
kann und die Komplexität des Zusammenspiels verschiedener automatisierter Prozesse und Systeme hier ergänzend zur Übersetzung von Implementation (Quellcode) zur Anwendung (Software) hinzukommt.

Nachfolgend sind einige beispielhafte Einblicke aufgelistet, die diesen Punkt verdeutlichen sollen. Es handelt sich um Kommentare im Quellcode sowie die soziale Auseinandersetzung und Aushandlung über Code im vorletzten Beispiel, bei dem ein Skript für einen beachtlichen Zeitraum von 1.000 Stunden gestoppt wird, wenn ein bestimmter Mitarbeiter als Administrator auf der Anwendungsebene aktiv ist.
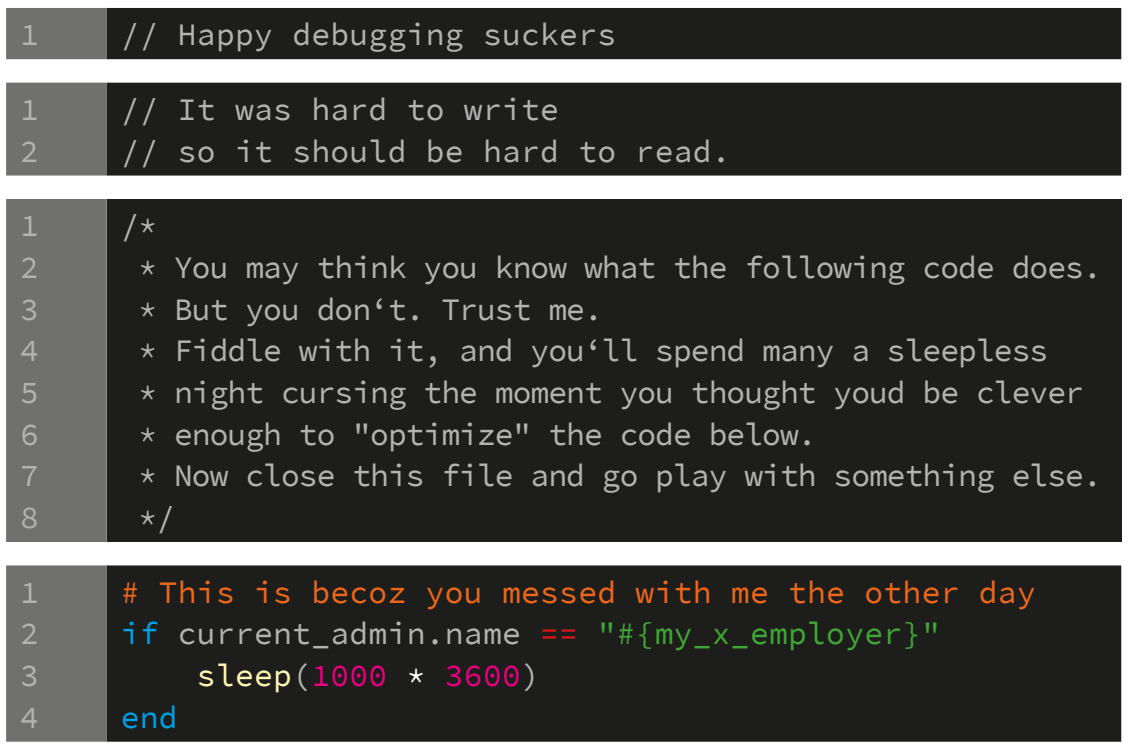

1 // they made me write it, against my will

Abb. 2.: Beispiele für Code Kommentare. Quelle: Eigene Darstellung.

Die reflexive Auseinandersetzung mit dem Gegenstand ist in technologischen Umgebungen keineswegs neu, sondern vielmehr ein fester Bestandteil, wie man am Beispiel von Gaming-Kulturen zeigen kann. So ist beispielsweise das Cheating nicht nur dadurch charakterisiert, dass man eine Aufgabe durch Zuhilfenahme 
externer Quellen oder einen Gegenspieler aufgrund einer den Rahmenbedingungen vorgesehenen Handlung überlistet, sondern dass man gleichzeitig die Rahmenbedingungen des Spiels verändert (vgl. Thiedeke 2007; Consalvo 2011). Gerade Wallhacks und Aimbots sind im Online-Gaming präsent und zugleich verpönt, sie basieren auf Skripten und Software, die dazu dienen, das vorherrschende Spielsystem zu unterwandern.

\section{Beobachtungen des Digitalen - Beobachtungen im Digitalen}

Was das für Soziale Medien bedeuten kann, lässt sich anhand des Microblogging-Dienstes Twitter beschreiben, dabei ist es zunächst ganz unerheblich, ob das Forschungsdesign ein qualitatives, quantitatives oder geprägt von Mixed Methods ist. Twitter als Unternehmen ist nicht zuletzt aufgrund der recht offenen Konzeption und des anschlussfähigen Umgangs mit den Daten durch die Bereitstellung einer umfangreichen Schnittstelle in den Fokus der Online-Forschung gerückt. Ein Tweet kann beispielsweise so aussehen:

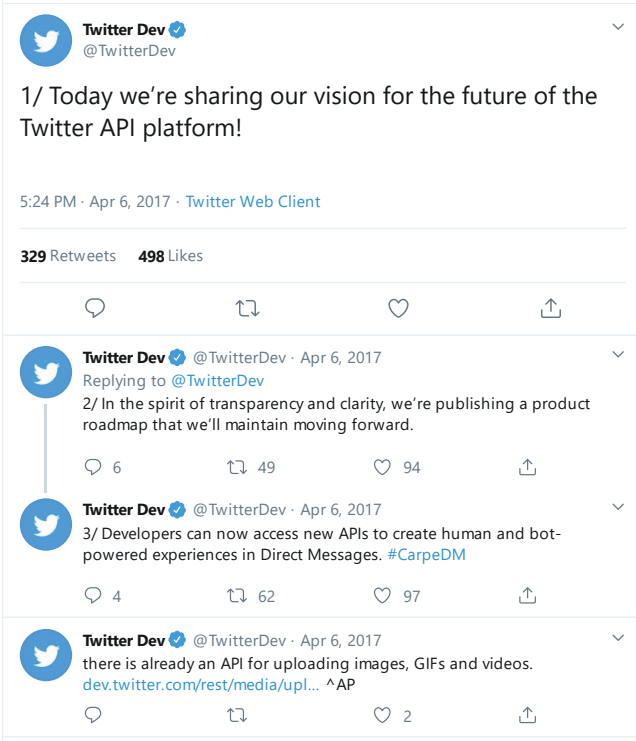

Abb. 3.: Darstellung eines Tweets auf Twitter.com. Quelle: Eigener Screenshot. 
Ein Tweet jedoch besteht aus mehr als nur maximal 280 Zeichen, da dem Tweet verschiedene Attribute, Beschreibungs- und Metadaten zugeordnet sind. Man kann beispielsweise die Geoposition oder Geräteinformationen bekommen und somit zu womöglich aussagekräftigerem Interpretationsmaterial gelangen auch mit Fokus auf die Online Forschung. Ein Tweet kann beispielsweise wie folgt aussehen:

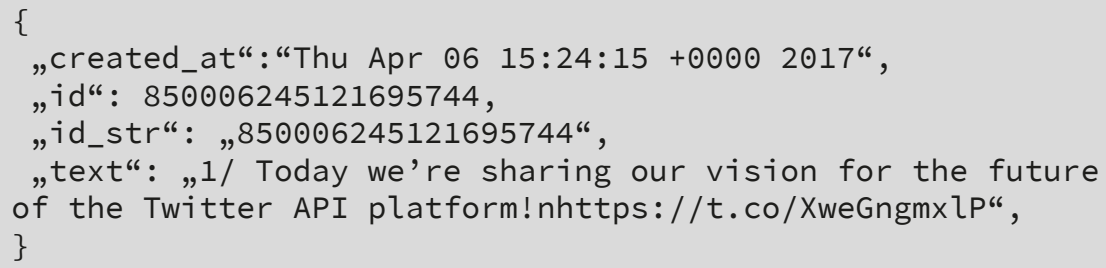

Hier werden lediglich das Erstellungsdatum, die ID und der Text im JSON-Format ausgegeben. Ergänzend dazu kann der Ort des Tweets als Attribut ebenfalls übergeben werden:

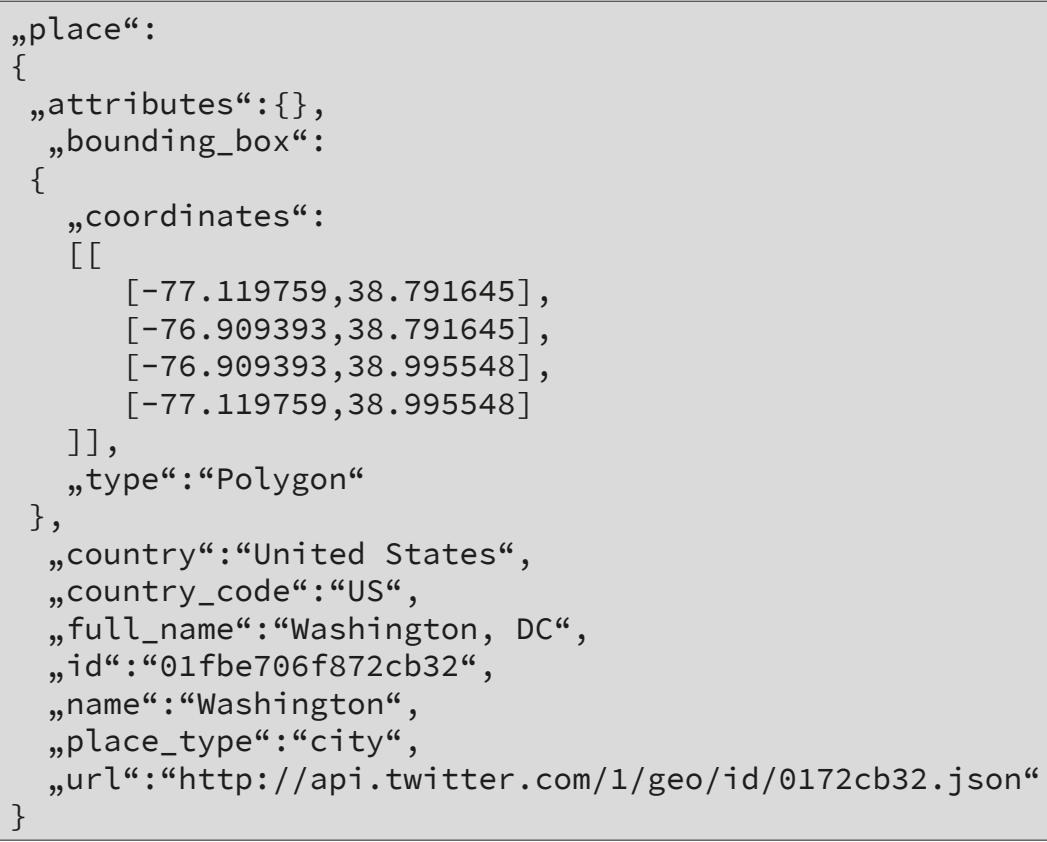


Es sind die digitalen Objekte und ihre Attribute, die uns die Daten so zusammenlegen, wie wir sie auf der Anwendungsoberfläche schliesslich sehen. Schnittstellen ermöglichen es verschiedenen Akteuren mit den Daten zu arbeiten und diese für eigene Vorhaben weiterzuverarbeiten.

\section{Digitale Methoden?}

Es steht ausser Frage, dass hier die kommerziellen Interessen nicht zuletzt von Twitter selbst - eine entscheidende Rolle spielen. Dennoch stellt die Öffnung durch die Schnittstellen eine Zugangsmöglichkeit zur Beobachtung und Rekontextualisierung dar. Diese Beobachtung kann unter Zuhilfenahme digitaler Methoden erfolgen und verbindet damit eine der zentralen Besonderheiten von Big Data, nämlich das Vorkommen qualitativer und quantitativer Daten zugleich. Die Medienpädagogik stellt hier wichtige Fragen, wie man beispielsweise durch die Arbeiten von Dander (2014) formuliert findet, wenn er den Weg von der «Macht der Daten» zur «Gemachtheit von Daten» beschreibt und damit auf eine lange Tradition machttheoretisch höchst relevanter Aushandlungsprozesse im Umgang mit Informationen und Wissen hinweist. Wie sich die Gemachtheit der Daten im spezifischen Feld verhält, kann empirisch erforscht werden, indem man eben jene Daten ins Verhältnis zueinander setzt, somit die Narrative des Sozialen entschlüsselt und schliesslich dahinter liegende Prozesse sichtbar macht.

Eingeräumt werden muss hier natürlich, dass derartige Forschungsvorhaben nur durch die Entwicklerschnittstellen erst möglich sind. Bis vor einiger Zeit konnte man derartige Analysen auch entlang von Facebook und verschiedenen individuellen Datensätzen machen. Die Anwendung Netvizz des Medienwissenschaftlers Rieder (2013) kann hier als Beispiel genannt werden, sie wurde jedoch vor dem Hintergrund der Debatte um personenbezogene Daten, eine mögliche De-Anonymisierung und den Schutz der individuellen Privatsphäre, sowie den Änderungen der allgemeinen 
Geschäftsbedingungen von Facebook um immer mehr Möglichkeiten gekürzt. Hier zeigt sich vor allem auch die Abhängigkeit zu Unternehmen, die diese Daten und den Zugang zu ihnen bereitstellen. Werden Rahmenbedingungen eines Angebots geändert, müssen digitale Analysewerkzeuge darauf reagieren. Wie die Daten gemacht werden, ist dann also nicht nur von den jeweiligen Akteuren im Anschluss an die Software abhängig, sondern man ist vor allem auch auf die Öffnung der Erbauer und Entwickler dieser Umgebungen angewiesen. Eine Möglichkeit des Umgangs mit möglichen Restriktionen bieten digitale Methoden dann, wenn man die Ausgaben und Ergebnisse, die geschlossene algorithmische Systeme liefern, im Sinne des Reverse Engineerings zurückverfolgt und dekonstruiert und dabei, Seaver (2017) folgend, vor allem auch kulturelle Implikationen und soziale Lagerungen sichtbar macht.

Durch eine Hinwendung zu Digital Methods können auch in einer erziehungswissenschaftlich orientierten Online-Forschung neue Perspektiven eingenommen werden, indem zunächst unsichtbare Gegenstände und Phänomene sichtbar gemacht werden können. Gleichzeitig sind sie ein Mittel der Befähigung, denn durch den Einsatz digitaler Werkzeuge, Techniken und Methoden können digitale Daten in einer besonderen Weise aufbereitet, dokumentiert und ins Verhältnis zueinander gesetzt werden.

Digitale Methoden und Werkzeuge können aber nicht nur genutzt werden, sondern gleichzeitig auch weiterentwickelt und erneut einem breiteren Kreis von Forschenden zugänglich gemacht werden. Dies kann beispielsweise über Open Source Repositorien, über SourceForge oder GitHub ${ }^{9}$ geschehen. Ein besonderes

9 Siehe hierzu auch https://sourceforge.net/ bzw. https://github.com/. Im Unterschied zum Online-Dienst SourceForge zur Verwaltung von Softwareprojekten steht bei GitHub nicht der Quelltext im Mittelpunkt des Konzepts, sondern die User mit ihren Repositories und Projektaktivitäten. Damit wird bei GitHub ein Fokus auf die soziale Interaktion, Projektkooperation und Teilnahme an fremden Projekten gelegt. 
Merkmal des Digitalen ist die Veränderbarkeit und der Rekontextualisierungsspielraum. Man kann also digitale Frameworks erweitern, verändern und diese für spätere Forschungsvorhaben anderen Interessenten bereitstellen. Damit wird das Digitale auch reflexiv im Sinne der Forschung gewendet. Zugleich können hierüber neue Erkenntnisse im Umgang mit Methoden und Methodologien gewonnen werden, da die Repositories gleichzeitig ein erster Schritt der Dokumentation sind. Das heisst gleichzeitig auch, dass digitale Methoden hinsichtlich der Leistungsfähigkeit geprüft werden müssen. Schliesslich sei ein zentrales Thema der Online-Forschung das «kritische Prüfen neuer Methoden und Instrumente» (Welker 2014, 23).

\section{Fazit und Ausblick}

Von der Ausgangsposition einer strukturalen Online-Ethnografie haben wir nun mit zwei Thesen auf einige Herausforderungen hinsichtlich methodischer und methodologischer Fragen im Umgang mit digitalen Daten hingewiesen, die wir hier zusammenfassen wollen. Unserer Diskussion lagen folgende Thesen zugrunde:

- Ethnografische und strukturale Elemente ermöglichen die Interpretation von Bedeutung und die Kartografierung heterogener digital-sozialer Kulturräume

- Digitale Daten in ihrer Qualität zu erfassen kann durch Reflexivität im Umgang mit dem Material gelingen

Die erste These verweist darauf, dass eine Ethnografie, die versucht soziale Organisation im Kontext einer digitalen Medialität zu verstehen, dies sowohl anhand der Teilnehmenden als auch anhand einer von Menschen gemachten sozio-technischen Umgebung tun sollte. Praxen, die zur Herstellung und Entwicklung einer OnlinePlattform führen, wie die Strukturmerkmale aus der Online-Ethnografie Marotzkis gezeigt haben, bilden ggf. eine neue Dynamik aus, weil sie bestimmte Nutzungen ermöglichen oder behindern könnten. 
Die zweite These zielt auf die wandelbaren Materialitäten des Digitalen ab, die als komplexe Simulationen von Materialität verstanden werden können:

«Das besondere - und historisch neue - Moment digitaler Medialität liegt auf dieser Ebene des Materiellen begründet: Digitalität de- und recodiert das materielle Moment von Mediatisierungsprozessen. Sie verdoppelt damit den oben dargestellten Prozess der Mediatisierung. Insofern bedeutet Digitalität eine 〈Mediatisierung von Mediatisierungen〉 (Mersch 2002, 69)» (Jörissen 2014, 505f.).

Dieses veränderte Verhältnis von Medialität verbunden mit einem bedeutenden Komplexitätszuwachs muss reflektiert werden und wirkt sich insofern auf den Modus von ethnografischer Methodologie aus, als dass sich Beteiligte dieses Verhältnisses nicht explizit bewusst sein müssen.

Beide Thesen stehen im engen Verhältnis zueinander, denn sie verweisen auf die tieferliegenden Implikationen gesellschaftlicher Veränderungsprozesse und sollen somit auf die Auswirkungen hindeuten, denen sich die Forschung in digitalen Räumen konfrontiert sieht. Beide Thesen dienen darüber hinaus als Aufforderung zur methodischen und methodologischen Reflexion ethnografischer Verfahren vor dem Hintergrund des Digitalen.

Eine Erweiterung der ethnografischen Perspektive auf die Infrastrukturen, die den digitalen Sphären zugrunde liegen, scheint uns hilfreich, um Prozesse und Phänomene sichtbar zu machen, die einer klassischen Perspektivsetzung zunächst womöglich verborgen bleiben könnten. Gemeint ist damit auch, die Entstehungskontexte der digitalen Daten in den Blick zu nehmen. Das heisst, dass OnlineForschung aus einer erziehungswissenschaftlichen oder medienpädagogischen Perspektive sich nicht nur auf die Anschlusspraktiken und Handlungsweisen im Vollzug beziehen kann, sondern auch den Blick «closer to the metal» wagen sollte. Ethnografische Verfahren 
bieten hier eine geeignete Möglichkeit, sich den Strukturen des Digitalen zuzuwenden.

\section{Literatur}

Berry, David M. 2011. The Philosophy of Software: Code and Mediation in the Digital Age. Basingstoke: Palgrave MacMillan.

Berry, David M., Hrsg. 2012. Understanding Digital Humanities. Houndmills, Basingstoke, Hampshire; New York: Palgrave Macmillan. https://doi.org/10.1057/9780230371934.

Brügger, Niels. 2011. «Web Archiving - Between Past, Present, and Future». In The Handbook of Internet Studies, herausgegeben von Mia Consalvo und Charles Ess, 24-42. Malden: Wiley-Blackwell.

Chun, Wendy H. K., Anna W. Fisher, und Thomas Keenan, Hrsg. 2015. New Media, Old Media: A History and Theory Reader. 2. Aufl. New York, London: Routledge.

Consalvo, Mia. 2009. Cheating: Gaining Advantage in Video Games. First MIT Press paperback edition. Cambridge, Mass: Massachusetts Institute of Technology.

Consalvo, Mia, und Charles Ess, Hrsg. 2011. The Handbook of Internet Studies (Handbooks in communication and media). Malden: Wiley-Blackwell.

Crawford, Kate. 2015. «Can an Algorithm Be Agonistic? Ten Scenes from Life in Calculated Publics». Science, Technology, \& Human Values 41 (1): 77-92.

Culkin, John M. 1967. «A Schoolman's Guide to Marshall McLuhan». Saturday Review 50 (11): 51-53.

Dander, Valentin. 2014. «Von der 〈Macht der Daten〉 zur 〈Gemachtheit von Daten〉. Praktische Datenkritik als Gegenstand der Medienpädagogik.» Mediale Kontrolle unter Beobachtung, (3). http://www. medialekontrolle.de/wp-content/uploads/2014/og/Dander-Valentin-2014-03-01.pdf.

Dutton, William H. 2014. The Oxford Handbook of Internet Studies. Oxford: Oxford Univ. Press.

Holze, Jens. 2017. «Digitales Wissen: bildungsrelevante Relationen zwischen Strukturen digitaler Medien und Konzepten von Wissen». Dissertation zur Erlangung des Dr. phil., Magdeburg: Otto-von-Guericke-Universität Magdeburg. 
Jörissen, Benjamin. 2014. «Digitale Medialität». In Handbuch Pädagogische Anthropologie, herausgegeben von Christoph Wulf und Jörg Zirfas, 503-13. Wiesbaden: Springer Fachmedien. https://oi. org/10.1007/978-3-531-18970-3_46.

Jörissen, Benjamin. 2016. «〈Digitale Bildung〉 und die Genealogie digitaler Kultur: historiographische Skizzen». MedienPädagogik: Zeitschrift für Theorie und Praxis der Medienbildung 25: 26-40. https://doi. org/10.21240/mpaed/25/2016.10.26.X.

Jörissen, Benjamin, und Winfried Marotzki. 2009. Medienbildung - eine Einführung: Theorie - Methoden - Analysen. Bad Heilbrunn: UTB.

Jörissen, Benjamin, und Dan Verständig. 2016. «Code, Software und Subjekt». In Das umkämpfte Netz: Macht- und medienbildungstheoretische Analysen zum Digitalen, herausgegeben von Ralf Biermann und Dan Verständig, 37-50. Wiesbaden: Springer Fachmedien.

Kozinets, Robert V. 2006. «Netnography». In The Sage Dictionary of Social Research Methods, herausgegeben von Victor Jupp, 193-95. London: Sage.

Kozinets, Robert V. 2010. Netnography: Doing ethnographic research online. London: Sage.

Lessig, Lawrence. 2010. Code: Version 2.0. New York: SoHo Books.

Logan, Robert K. 2013. McLuhan Misunderstood: Setting the Record Straight. Toronto: Key Publishing House Incorporated. [Kindle Edition].

Marotzki, Winfried. 2003. «Online-Ethnographie - Wege und Ergebnisse zur Forschung im Kulturraum Internet». In Jahrbuch Medienpädagogik 3, herausgegeben von Ben Bachmair, Peter Diepold, und Claudia De Witt. Leverkusen: Leske + Budrich.

Marotzki, Winfried. 2008. «Internet Communities». In Grundbegriffe Ganztagsbildung: Das Handbuch, herausgegeben von Thomas Coelen und Hans-Uwe Otto, 402-10. Wiesbaden: VS Verlag für Sozialwissenschaften. https://doi.org/10.1007/978-3-531-91161-8_40.

McLuhan, Marshall. 1969. Counterblast. Toronto: McClelland and Stewart.

McLuhan, Marshall. 1992. Die magischen Kanäle. Understanding Media. Düsseldorf: Econ.

McLuhan, Marshall, und Quentin Fiore. 1967. Medium Is the Massage: An inventory of effects. 1. Aufl. London: Penguin Books.

McLuhan, Marshall, und Eric McLuhan. 1988. Laws of Media. Toronto: University of Toronto Press. 
Rieder, Bernhard. 2013. «Studying Facebook via data extraction: the Netvizz application». In WebSci '13 Proceedings of the 5th Annual ACM Web Science Conference, 346-55. New York: ACM.

Seaver, Nick. 2017. «Algorithms as culture: Some tactics for the ethnography of algorithmic systems». Big Data \& Society 4 (2): 2053951717738104. https://doi.org/10.1177/2053951717738104.

Stalder, Felix. 2016. Kultur der Digitalität. Frankfurt am Main: Suhrkamp.

Thiedeke, Udo. 2007. "Trust, but test!: das Vertrauen in virtuellen Gemeinschaften». Zugl.: Mainz, Univ., Habil.-Schr., Konstanz: UVK Verlagsgesellschaft. http://deposit.d-nb.de/cgi-bin/ dokserv?id=2901059\&prov=M\&dok_var=1\&dok_ext=htm.

Verständig, Dan. 2017. «Bildung und Öffentlichkeit - Eine strukturtheoretische Perspektive auf Bildung im Horizont digitaler Medialität». Dissertation, Magdeburg. http://nbn-resolving.de/ urn:nbn:de:gbv:ma9:1-1009o.

Verständig, Dan, und Jens Holze. 2016. «It's not just a game - Subversive Praktiken in digitalen Spielkulturen». In Phänomen Let's Play-Video: Entstehung, Ästhetik, Aneignung und Faszination aufgezeichneten Computerspielhandelns, herausgegeben von Judith Ackermann, 225-39. Wiesbaden: Springer Fachmedien. https://doi.org/10.1007/978-3-65812936-1_16.

Welker, Martin. 2014. «Normalisierung und Ausdifferenzierung von Online-Forschung - Eine Einführung». In Handbuch Online-Forschung: Sozialwissenschaftliche Datengewinnung und -auswertung in digitalen Netzen, herausgegeben von Martin Welker, Monika Taddicken, JanHinrik Schmidt, und Nikolaus Jackob, 14-41. Köln: Herbert von Halem Verlag.

Wellman, Barry. 2004. «The Three Ages of Internet Studies: Ten, Five and Zero Years Ago». New media \& society 6 (1). http://homes.chass. utoronto.ca/ tkennedy/Courses/2P26/Wellman\%202004.pdf. 